\title{
A Multipath Energy-Efficient Probability Routing Protocol in Ad Hoc Networks
}

\author{
Zhimu Huang*, Ryo Yamamoto*, Yoshiaki Tanaka**** \\ *Global Information and Telecommunication Institute, Waseda University \\ 1-3-10 Nishi-Waseda, Shinjuku-ku, Tokyo, 169-0051 Japan \\ **Research Institute for Science and Engineering, Waseda University \\ 17 Kikuicho, Shinjuku-ku, Tokyo, 162-0044 Japan
}

zhimu_h@fuji.waseda.jp,ryo_yamamoto@moegi.waseda.jp,ytanaka@waseda,jp

\begin{abstract}
Ad hoc networks are decentralized type of wireless networks. Moreover, ad hoc networks are characterized by random, multi-hop topologies that may change rapidly over time because of mobile nodes. However, since nodes in ad hoc networks operate on limited battery energy and it is impractical to recharge or replace the battery, an energy-efficient protocol is important in the design of ad hoc networks. By conventional routing protocols, a shortest path is always selected in ad hoc networks. Without considering the energy consumption, some nodes will exhaust very soon and ad hoc networks will become partitioned. Improving routing protocols to prolong the lifetime of ad hoc networks has been a hot research area in the past few years. However, most of protocols only focus on the constrained battery energy. In this paper, a new protocol is proposed. It is a multipath energy-efficient probability routing protocol based on AODV (MEP-AODV). In MEP-AODV, not only battery energy consumption but also multipath selection is considered. By the proposed protocol, when an intermediate node received a request packet (RREQ), it won't relay the RREQ immediately. It will relay the RREQ with a probability which is based on its remaining battery energy. After the RREQ arrived at the destination node, it won't trigger a reply packet (RREP) at once until the expiry of a delay timer. The destination will select multiple paths with sufficient battery energy from the collected paths after the timer expired. Then the destination node initiates the corresponding RREPs. The source node can send data packets via the selected multiple paths by a probability function which is based on the minimum node remaining battery energy of one path. This proposed protocol is implemented in QualNet to evaluate the performance. From the results, MEP-AODV shows the good energy efficiency in terms of the maximizing the lifetime of ad hoc networks.
\end{abstract}

Keywords-MEP, AODV, Multipath, Routing, Energy, Lifetime, Ad hoc networks

\section{INTRODUCTION}

Ad hoc networks are flexible and decentralized wireless networks. Ad hoc networks do not rely on any fixed and preexisting infrastructure such as routers in wired networks, access points in wireless networks and base station in cellular networks. Instead of this, all the nodes in ad hoc networks can act as either routers or terminals, or sometimes both of them. The determination of which role nodes act as is made dynamically just based on a connectivity of networks. Thus, each node in ad hoc networks is equal. Moreover, it can be free for each node to associate with any other nodes in the network in radio range for building communications. Ad hoc networks are characterized as self-organized networks and mobility. With these outstanding characteristics, ad hoc networks have attracted a lot of attention and have been implemented in many tactic networks related to the applications such as disaster discovery and relief, battlefield monitoring and communications, surveillance and law enforcement.

All the nodes in ad hoc networks use battery as power, however, it is impractical to recharge or replace the battery when they exhaust their battery energy. Therefore, ad hoc networks are energy-constraint and it is crucial to design an energy-efficient protocol in ad hoc networks. Conventional routing protocols such as Ad hoc On Demand Distance Vector (AODV) and Dynamic Source Routing (DSR) do not consider energy consumption as a design constraint. Shortest-path algorithms which usually have the minimum hop count are always used and routes would not be modified until they are disconnected. Thus, ad hoc networks may become partitioned once some nodes drain their battery energy and die earlier than others. As concerns the problem, developing energyefficient routing protocols for ad hoc networks has been a popular research area during the past few years. Many proposals focus on how to minimize energy consumption or how to balance energy consumption to prevent such premature death. However, these proposals to the energy-efficient problem mostly consider one-path routing algorithms.

This paper presents a novel multipath routing algorithm aiming to improve the existing routing protocol more efficiently through a multipath energy-efficient probability routing protocol (MEP-AODV) which is based on AODV. In order to select the optimal paths with the sufficient battery energy, each RREQ will be transmitted with a probability which is based the remaining battery energy of one node and a multipath routing algorithm is considered. The remainder of this paper is organized as follows. In section II, we describe a review of related work. In section III, a new routing protocol MEP-AODV is introduced. Section IV shows the network simulation results and the performance evaluation. Finally, section $\mathrm{V}$ concludes this paper. 


\section{RELATED WORKS}

Energy-efficient routing protocols can be classified into 3 categories: idle energy save protocols, transmission power control protocols and energy-aware routing protocols.

\section{A. Idle Energy Save Protocols}

In idle energy save protocols [1], [2], idle nodes can be turned to sleeping mode. This is because that radio utilized for communication can consume energy not only when receiving or transmitting, but also when idle [3]. Thus, energy can be conserved by turning idle nodes to sleeping mode. There are different strategies to determine what time nodes should turn to sleeping mode and what time to wake up.

\section{B. Transmission Power Control Protocols}

About transmission power control protocol [4]-[6], transmission power can significantly impact transmission range in ad hoc networks. Traditionally, transmission power is fixed during the entire communication process. As the transmission power of one node is adjusted, the node can select its neighbours and the network topology changes accordingly. In [4], a dynamic power adjustment approach is proposed. In this way, transmission power can be decreased. However, decreasing the transmission power would cause an increase of error rate of one frame and it is can be compensated by retransmission. Since not all frames transmitted at the decreased transmission power need to be retransmitted, the actual total transmission energy consumption can be potentially less than the energy consumption by the original fixed transmission power scheme.

\section{Energy-Aware Routing Protocols}

As mentioned above, idle energy save protocols and transmission power control protocols can reduce total energy consumption effectively. However, both of them could not solve the problem since some nodes die earlier which are used often, and routes become unavailable and even the whole network becomes lack of connectivity between nodes that are still alive.

As concern this problem, many energy-aware routing protocols are proposed. In ad hoc networks, routing protocols can be categorized into two groups. One is proactive routing protocol and the other is reactive routing protocols. In proactive routing protocols, each node maintains one or more route tables for the entire network. A change in topology would be propagated through all the nodes in the network and all the nodes have to update the tables. In reactive routing protocols, a route to destination is established only when it is necessary. Compared to proactive protocols, reactive routing protocols generate less routing overhead and less energy consumption. Thus, many proposals to energy-efficient routing protocols are based on reactive routing protocols.

The majority of these protocols focus on two separate issues. One is selecting the route with the minimal total transmission power during the delivery of one packet and the other is maximizing the lifetime of ad hoc networks.

In [7], minimum total transmission power routing (MTPR) is described. In the paper, the total transmission power for route $r P_{r}$ is derived by Eq.(1):

$$
P_{r}=P\left(n_{s}, n_{d}\right)=\sum_{l_{a} \in r} P\left(l_{a}\right)
$$

where $n_{s}$ and $n_{d 1}$ are the source node and the destination node, respectively. $P\left(l_{a}\right)$ is the transmission power between link $l_{a}$ in route $r . P\left(n_{s}, n_{d}\right)$ is the transmission power between $n_{s}$ and $n_{d} . P\left(n_{s}, n_{d}\right)$ is not fixed and can be adjusted. Therefore, the desired route with the minimum total transmission power $P_{k}$ can be given by Eq.(2):

$$
P_{k}=\min \left\{P_{r} \mid r \in A\right\}
$$

where $A$ is a set containing all possible routes. Transmission power is always fixed during the entire communication process. However, in MTPR, transmission power can be adjusted based on the distance between two nodes. Transmission power is proportional to $d^{n}$, where $d$ is the distance between $n_{s}$ and $n_{d}$, and usually $m$ is equal to 2 or 4 . $P_{r}$ with the shorter distance between two neighbouring nodes may be smaller. The shorter distance usually means that more hops will be more likely to involve in transmitting packets. The end to end delay will increase. In addition, a route with more nodes is more likely to be unstable since the intermediate nodes are more likely to move away from the route. Selecting the route with the minimal total transmission power can reduce the total transmission energy. However, it does not prolong the lifetime of ad hoc networks in some cases. We can assume that the minimal total transmission power routes always go through a special node and the node would exhaust its battery energy more quickly and the routes will become unavailable. Therefore, how to maximize the lifetime of ad hoc networks become a crucial issue and the remaining battery energy of each node becomes a more accurate metric to describe the lifetime of each node.

In [8], min-max battery cost routing (MMBCR) is described. To make sure that each node is not overused, let $c_{i}$ be the remaining battery energy of node $i$. In the paper, $f(i)$ is defined as the node cost which can be given by Eq.(3):

$$
f(i)=\frac{1}{C_{i}}
$$

This means that the less remaining battery energy is, the more the node cost will be. The minimum route cost function is obtained from Eqs.(4) and (5):

$$
\begin{aligned}
& R_{j}=\max \{f(i)\} \\
& R_{i}=\min \left\{R_{j} \mid j \in A\right\}
\end{aligned}
$$

where node $i$ is included in route $j$ and $R_{j}$ is the route cost of route $j . \quad R_{j}$ also means the minimum remaining node battery energy of route $j . \quad A$ is the set containing all the possible routes. $R_{i}$ is the minimum route cost. In this consequence, a route with $R_{i}$ will be selected. In MMBCR, some special nodes that may be used often are always considered. Thus, MMBCR can tries to avoid selecting the route, which passes through the nodes having a little battery energy. In MMBCR, the battery energy of each node is used more balanced and the lifetime of ad hoc networks seems to be prolonged. However, it is not guaranteed that the route with $R_{i}$ is always the shortest 
path under all the cases. If the route has more hops than shortest path, more energy would be consumed. Meanwhile, since $R_{i}$ is the minimum value among all the $R_{j} \mathrm{~s}$, all the possible route information must be collected and that would give the whole network tremendous traffic load.

In [9], another method is presented. The proposed energyaware probability routing mechanism (EAPR) uses a probability function to determine whether relay or drop the RREQ during the route discovery process. The function can be defined by Eq.(6):

$$
p_{i}= \begin{cases}1 & c_{i} \geq \theta \\ \frac{\alpha c_{i}}{\theta} & c_{i}<\theta\end{cases}
$$

where $c_{i}$ is the remaining battery energy, $\theta$ is the predefined threshold which is fixed for all the nodes and $\alpha$ is a coefficient. If $c_{i}$ is more than $\theta$, this RREQ would be processed and relayed. Otherwise, the RREQ would be dropped or is processed depends on $p_{i}$ determined by the remaining battery energy of node $i$. If one node has the lower remaining battery energy, the node has the smaller possibility to participate in the route discovery process. Thus, it greatly reduces the traffic load in the broadcasting RREQ process. However, a route with a node that has lower remaining battery energy is still likely to be selected since this node still has a probability $1-p_{i}$ to relay an RREQ.

As described above, many methods to solve the energyefficient issue focus on one-path routing algorithms. If multipath routing algorithms, which can acquire multiple routes during one route discovery process, are used, it will reduce the re-initiation of the route discovery process. Multiple alternative routes are used and can be switched for transmitting data packets. Thus, comparing the algorithms which always use one route to transmit data packets, it is expected to have a better performance in terms of maximizing the lifetime of ad hoc networks.

\section{Multipath ENERgY-EfFicient PROBABILITy Routing PRotocol (MEP-AODV)}

In this section, in order to maximize the lifetime of ad hoc network, we propose a novel routing protocol named MEPAODV, which is an extension of AODV.

\section{A. Overview of AODV}

AODV is a reactive routing protocol which is often used in ad hoc networks. In AODV, nodes maintain routes only when they are needed. One source node has some data to send to a destination node with no available route, a route discovery process is initiated. In the route discovery process, the source node broadcasts route request (RREQ) toward the destination node. When an intermediate node receives the RREQ, it checks whether the RREQ was received before or not using a sequence number inside the RREQ. If not, the RREQ is rebroadcasted to other nodes and a reverse path to the source node is established with the previous hop as the next hop on the reverse route. When the first RREQ arrives at the destination node, a route reply (RREP) is generated immediately and is sent back to the source node using unicast through the established reverse path. If other RREQs are arrived at the destination node after the node send back RREP, the RREQs will be discarded. As this RREP is forwarded to the source node, a route for the data transmission is established. If an intermediate node has available route to the destination, the node immediately send back RREP instead of the destination terminal. When a link failure is detected, a route error packet (RERR) is sent back to all the source nodes using the route. After erasing this route, a new route discovery process is initiated if one route to the destination node still be needed.

\section{B. Probability Function in MEP-AODV}

In MEP-AODV, the remaining battery energy of all the nodes except the source node and the destination node is taken into account for balancing the energy consumption.

When an intermediate node receives an RREQ, it checks its remaining battery energy and to determines whether relay this RREQ or drop it by a probability function which can be given by Eqs.(7) and (8):

$$
\begin{aligned}
b_{i} & =\frac{c_{i}}{e_{i}} \\
p_{i} & = \begin{cases}1 & b_{i} \geq r_{1} \\
1-\frac{r_{1}-b_{i}}{r_{1}-r_{2}} & r_{2} \leq b_{i}<r_{1} \\
0 & b_{i}<r_{2}\end{cases}
\end{aligned}
$$

where $c_{i}$ is the current remaining battery energy of node $i$ and $e_{i}$ is the full battery energy of node $i . b_{i}$ is the remaining energy ration of $c_{i}$. $r_{1}$ and $r_{2}$ are predefined thresholds and the values are $1>r_{1}>r_{2}>0 . \quad p_{i}$ represents the probability of RREQ rebroadcasting. When $b_{i}$ is more than $r_{1}$, it means that node $i$ has sufficient remaining battery energy and definitely relays the RREQ. When $b_{i}$ is between $r_{1}$ and $r_{2}$, the probability relaying the RREQ only depends on $b_{i}$. When $b_{i}$ is less than $r_{2}$, it means the node has very low battery energy and need to be protected. Thus, this RREQ will be discarded. Then, the node turns to idle mode. This is because that even if the node cannot transmit any packets, it will still consume energy which is less than transmitting or receiving a packet.

\section{Route Discovery Process in MEP-AODV}

In order to build MEP-AODV, we modify RREQ and RREP packet. One new parameter $b_{l}$ is added in the header of RREQ and RREP. $b_{l}$ describes the minimum node remaining battery energy of route $l$. When one source node requires a route to a destination node for sending data packets, it checks its route table. If there is no available route to the destination, the route discovery process is initiated and RREQ is flooded to neighbouring nodes.

When an intermediate node receives an RREQ, it checks whether the RREQ was preceded before or not. If not, next phase can begin. Otherwise, the RREQ would be discarded. After the RREQ is confirmed as the one that is the first time to 
be received, the node checks its remaining battery energy $c_{i}$ to determine whether process the RREQ by the probability function as defined in Eq.(8). If it determines to process this RREQ, the ratio of the remaining battery energy $b_{i}$ is compared with $b_{l}$. If $b_{i}$ is less than $b_{l}, b_{l}$ would be replaced by $b_{i}$. Then the RREQ is rebroadcasted toward the destination node. This process is repeated until the RREQ arrives at the destination node [10].

When the destination node receives the first RREQ, it does not generate a RREP and send it back to the source node immediately. The received RREQs would be buffered for a certain period until delay timer expires in order to collect as many RREQs as possible. Note that the delay timer should not be set too short or too long. If it is too short, the destination node cannot collect enough RREQs. If it is set too long, that increases the end to end delay. Since we consider the end to end delay, collecting all the possible routes is not necessary. During the period of the delay timer, RREQs which have relatively less time cost from the source node to the destination node are more likely to arrive at the destination node. It also means these routes which the RREQs passed through have relatively less hop count. After the expiry of delay timer, the destination node compares $b_{l}$ among the collected RREQs. In the procedure, the destination node selects $k$ paths which have the sufficient remaining battery energy. $k$ also should not be too large since too many alternative routes do not make more effect on the performance. On the contrary, maintaining too many routes generates more overhead [11]. After the route selection, the destination node initiates the corresponding RREPs and unicasts them through the reverse path. In the header of each RREP, $b_{l}$ is added.

When the source node receives a RREP, a route to the destination node is established and the RREP is buffered. After the $k$-th RREP arrives at the source node, $b_{l}$, which is in the header of each RREP, is loaded. A probability function for route selection to transmit data packets can be obtained by Eq.(9):

$$
p_{r}=\frac{b_{l}(i)}{\sum_{i=1}^{k} b_{l}(i)}
$$

where $p_{r}$ is the probability for selecting a route and $k$ is the number of the multiple routes. The larger $b_{l}$ one route has, the more possible to be selected as a transmission route. The multiple routes will be switched according to their probability when the source node needs to transmit a data packet. This process is repeated until a new route discovery process is initiated.

Figure1 shows an example. $b$ is the remaining energy ratio. Node $\mathrm{S}$ is the source node. It initiates a route discovery process. When an RREQ arrives at an intermediate node, it will check whether this is a duplicate RREQ or not. If not, this RREQ would be processed. We can assume that $r_{1}$ is equal to 0.5 and $r_{2}$ is equal to 0.2 . When node I receives an RREQ from node $\mathrm{H}$, it will relay this RREQ with a probability 0.33 since it has no sufficient remaining battery energy given by Eq.(8). However, this node has a probability 0.67 to drop

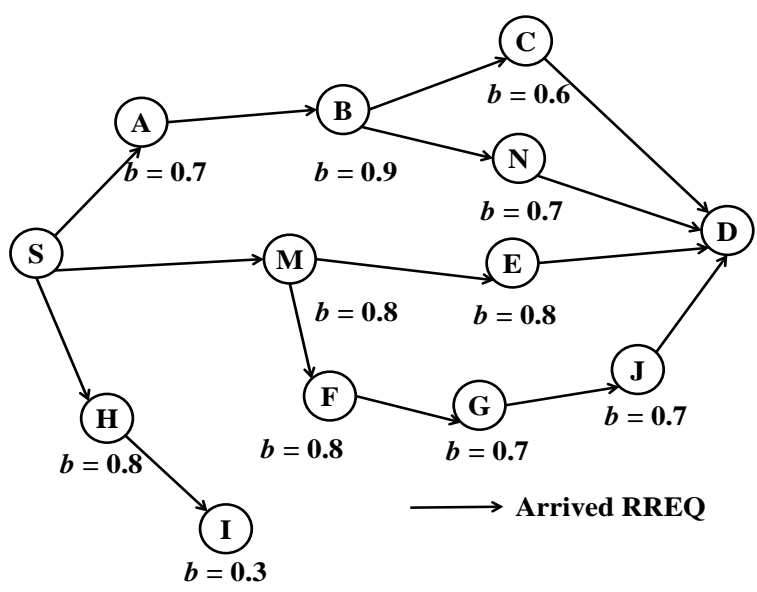

(a) Flooding of RREQ

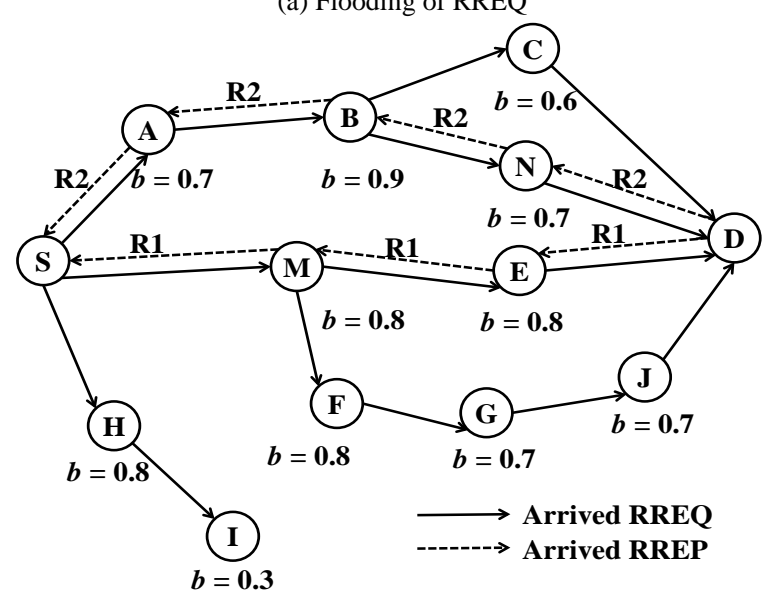

(b) RREP replying using multiple reverse path

Figure 1. Route discovery process MEP-AODV

this RREQ. In this figure, we assume that the node does not process the RREQ. However, it may have a probability to participate in the route discovery process for the next route discovery process. Node $\mathrm{D}$ is the destination node and we assume that node $\mathrm{D}$ receives three RREQs from node $\mathrm{C}, \mathrm{N}$ and $\mathrm{E}$ before the expiry of a delay timer. The RREQ from node $\mathrm{J}$ arrives after the delay timer expiration and it is discarded. Assume that $k$ is equal to 2, node $\mathrm{D}$ will choose two paths, D-E-M-S and D-N-B-A-S since the minimum $b$ of the route is 0.7 and 0.8 , respectively. Then node $\mathrm{D}$ sends the corresponding RREPs to node $\mathrm{S}$. When node $\mathrm{S}$ receives these two RREPs, two alternative routes R1 and R2 are established. R1 and R2 would be continually switched by using the probability defined by Eq.(9) when node $S$ transmits data packets. The probability of selecting R1 and R2 is 0.47 and 0.53 , respectively. When node $\mathrm{S}$ wants to send a data packet, $\mathrm{R} 1$ is select to transmit the packet with the probability of 0.53 . Otherwise, R2 is selected.

\section{Route Maintenance}

During a delivery of a large number of data packets, the remaining battery energy of some nodes may be consumed a lot. As concern this problem, in our protocol, intermediate 
nodes periodically check their remaining battery energy. If the status of some nodes becomes worse, they initiate an RERR and send it to the source node. When the intermediate node receives the RERR, it removes the corresponding item from its route table. Then, it brings the source node to rebuild a new route by initiating a route discovery process when this RERR arrives at the source node if the source node still remains some data packets which needs to be sent to the destination node. As well as AODV, the proposed MEPAODV also utilizes Hello messages to monitor the connectivity of ad hoc networks and confirm whether the routes are available or not. Neighbouring nodes can periodically initiate Hello messages and exchange them with each other. The absence of Hello messages indicates the failure of link connectivity. If a link failure is detected, this node removes the corresponding item from its route table. Then it initiates an RERR and sends it back to the source node and a new route discovery process is initiated.

\section{IV.PERFormanCE EVAluATion}

To evaluate the performance of MEP-AODV, we use QualNet 6.1 [12] as a simulator. Meanwhile, AODV and EAPR are chosen as the yardsticks for comparison.

\section{A. Overview of AODV}

In the simulation, we randomly distributed 50 nodes in $1500 \mathrm{~m} \times 1500 \mathrm{~m}$ area. The simulation time is $1000 \mathrm{~s}$. In order to achieve an energy-efficient protocol, it is crucial to use an energy model. In the simulation, we use a user defined energy model. When a node transmits or receives a packet, it will consume $800 \mathrm{~mA}$ per second or $650 \mathrm{~mA}$ per second, respectively. When the node is in the idle mode, it consumes $45 \mathrm{~mA}$ per second. About the battery model, we use a linear battery model, where the initial battery capacity is 30mAh for each node. In the network, the random waypoint model is used. In this model, each node can select a random destination. Then, the node moves to the destination and pauses for a fixed period with a random speed. After this period, the node selects another random location and move again. Each node repeats this process until the simulation time expires. In the simulation, we set the pause time to $10 \mathrm{~s}$, the minimum speed to $0 \mathrm{~m} / \mathrm{s}$ and the maximum speed to $2.5 \mathrm{~m} / \mathrm{s}$, $5 \mathrm{~m} / \mathrm{s}, 7.5 \mathrm{~m} / \mathrm{s}$ and $10 \mathrm{~m} / \mathrm{s}$. When the maximum speed is $0 \mathrm{~m} / \mathrm{s}$, it means this is a static network. We use CBR and fix the traffic load at 5 packets per second. In MEP-AODV, the source node can use three routes $(k=3)$.

\section{B. Performance Evaluation Metrics}

The most important metric is the network lifetime. The network lifetime can be defined in some ways. It can be the time taken for the first node to exhaust its battery energy or the time taken for all the nodes in the network to exhaust their battery energy. In the simulation, we adopt the definition former one. This metric can describe the lifespan of ad hoc networks.

Packet delivery ratio is the ratio of the total number of data packets received at the destination node to the total number of data packets transmitted by the source node. By this metric, we can see whether routes are stable or not.

Average energy consumption is defined that the total battery energy consumption in the network is divided by the total number of nodes. By it, we can see the energy-saving effect in ad hoc networks.

We define the average time between the moment a route discovery process is initiated by a source node and the moment a destination node receives the first data packet as end to end delay. This metric can present the quality of service in ad hoc networks.

\section{Simulation Results}

Figure 2 shows the simulation results of network lifetime with different maximum moving speed. The proposed MEPAODV has the best performance. When comparing with AODV, the proposed method increases the network lifetime about $11 \%$ when the moving speed is $7.5 \mathrm{~m} / \mathrm{s}$. Comparing with EAPR, MEP-AODV achieved 8\% lifetime increment. This is because routes are selected based on difference of battery energy level of nodes and routes with the insufficient battery energy can be avoided in MEP-AODV. Moreover, multipath routing algorithm enables balanced routing in terms of the battery energy in the network. Thus, MEP-AODV outperforms EAPR and AODV.

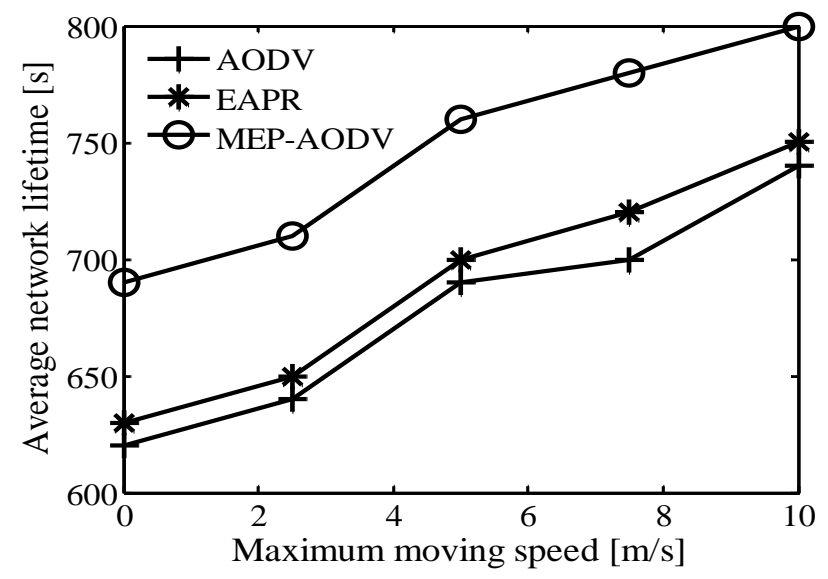

Figure 2. Average network lifetime

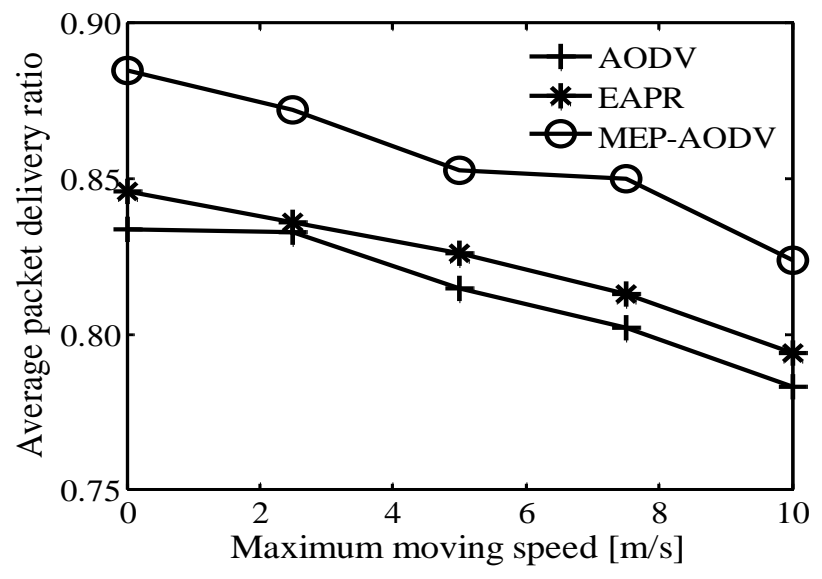

Figure 3. Average packet delivery ratio 


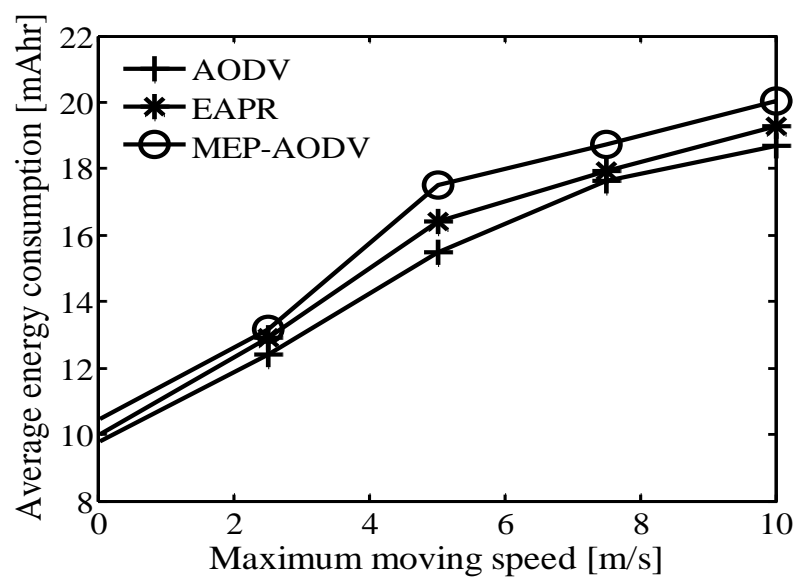

Figure 4. Average energy consumption

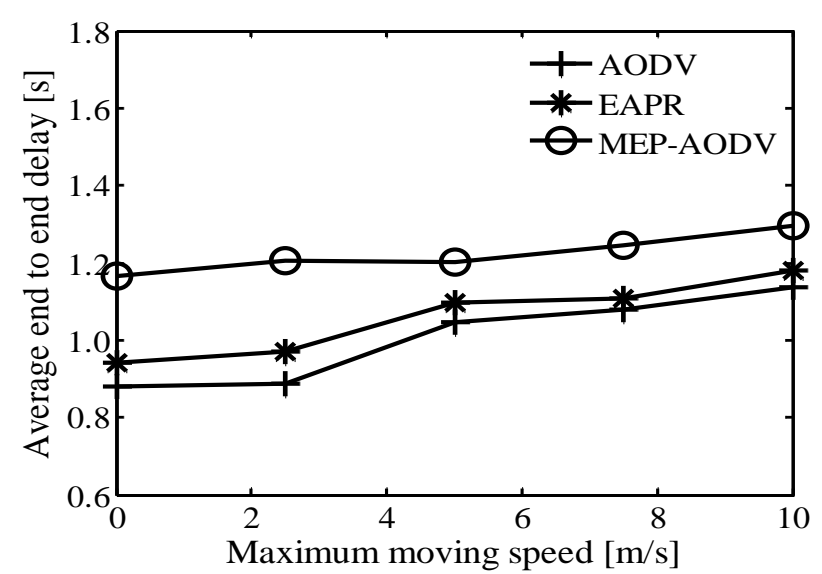

Figure 5. Average end to end delay

As shown in Figure 3, the average data packet delivery ratio in MEP-AODV is more than EAPR and AODV. In MEP-AODV, instead of the shortest path algorithms, battery energy consumption is always considered and the selected routes with sufficient battery energy usually have longer hop count. Increasing hop count may shorten the distance between two nodes. This makes packets transmission more stable than longer distance transmission. Moreover, in MEP-AODV, the routes with sufficient battery energy are selected and the multipath routing algorithm is considered. It decreases the possibility of link failure. Thus, less data packets are dropped due to a route disconnection.

Figure 4 shows the average energy consumption of nodes. In MER-AODV, the total energy consumption seems to be more than EAPR and AODV since it selects slightly longer routes than they do. However, in EAPR and AODV, the data packets which fail to the destination node also consumes energy. Meanwhile, MER-AODV decreases the battery energy consumption by minimizing re-initiation of route discovery process due to route disconnection. Consequently, as shown in Figure 4, MEP-AODV has a better performance.

Figure 5 shows the end to end delay. From this figure we can see MEP-AODV has no good performance, comparing with AODV and EASR. Since in MEP-AODV, it is not guaranteed that a shortest route is used and usually, MEPAODV has longer routes. Moreover, when a destination selects multiple routes, a delay timer is used. Thus in MEPAODV, the end to end delay increases. However, a little longer end to end delay does not affect improving the lifetime of ad hoc network.

\section{CONCLUSiOnS}

In this paper, a new routing protocol, MEP-AODV, is proposed. It can achieve a better performance in terms of prolonging the lifetime of ad hoc networks. MEP-AODV selects multiple routes with the sufficient battery energy and it avoids selecting the route with low battery energy node. By this method, the battery energy of each node is used more balanced. The source node can select one route from multiple alternative routes to send data packets and this can decrease the re-initiation of route discovery process. From the simulation results, the proposed MEP-AODV improves the performance in terms of network lifetime, packet delivery ratio and average energy consumption comparing with EAPR and AODV. The result shows that limited battery energy in ad hoc networks can be utilized more efficiently. However, in order to select the routes with sufficient battery energy, MEPAODV increases an end to end delay of data packets transmission since it utilizes longer routes and waiting time of a delay timer. In the future work, how to minimize the end to end delay should be considered.

\section{REFERENCES}

[1] S. Singh and C.S. Raghavendra, "Power efficient MAC protocol for multi-hop radio networks,” in Proc. IEEE Int. Symp. Personal, Indoor and Mobile Radio Commun., vol. 1, pp. 153-157, Sept. 1998.

[2] Y. Xu, J.Heidemann, and D.Estrin, “Adaptive energy conserving routing for multihop ad hoc networks," Research Report of USC/Information Sciences Institute, Oct. 2000.

[3] J. Li, D. Cordes, and J. Zhang, "Power-aware routing protocols in ad hoc wireless networks,” in Proc. IEEE Conf. Wireless Commun., vol. 12, no. 6, pp. 69-81, Dec. 2005.

[4] Y. Zhao, and M.S. Hsiao, "Reducing power consumption by utilizing retransmission in short range wireless network," in Proc. IEEE Conf. Local Comput. Netw. (LCN), pp. 527-533, Nov. 2002.

[5] L. Hu, "Topology control for multihop packet radio networks," IEEE Trans. Commun., vol. 41, no. 10, pp. 1474-1481, Oct. 1993.

[6] R. Ramanathan and R. Rosales-Hain, "Topology control of multihop wireless networks using transmit power adjustment," in Proc. 9th Annual Joint Conf. of IEEE Comput. And Commun. Societies (INFOCOM), vol. 2, pp. 404-413, March 2000.

[7] C-K. Toh, "Maximum battery life routing to support ubiquitous mobile computing in wireless ad hoc networks,” IEEE Commun. Mag., vol. 39, no. 6, pp. 138-147, June 2001.

[8] S. Singh, M. Woo, and C.S. Raghavendra, "Power-aware routing in mobile ad hoc networks," in Proc. 4th Annual ACM/IEEE Int. Conf. Mobile Comput. Netw., pp. 181-190, Oct. 1998.

[9] L. Cao, T. Dahlberg, and Y. Wang, "Performance evaluation of energy efficient ad hoc routing protocols," in Proc. IEEE Int. Conf. Performance, Comput., Commun., pp. 306-313, April 2007.

[10] C.T. Cheng, C.K. Tse and F.C.M. Lau, "An Energy-Aware Scheduling Scheme for Wireless Sensor Networks,” IEEE Trans. Vehicular Tech., vol. 59, no. 7, pp. 3427-3444, Sept. 2010.

[11] W. Cho, D. Kim, and T. Kim, "Time delay on-demand multipath routing protocol in mobile ad-hoc networks," in Proc. Int Conf. Ubiquitous and Future Netw., pp.55-60, June 2011.

[12] (2002) The Scalable network technologies website. [Online]. Available: http://web.scalable-networks.com/ 


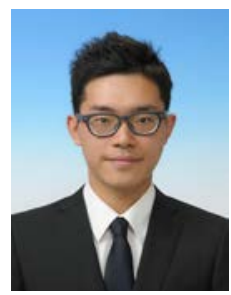

Zhimu Huang received his B. E. degree in communication science and engineering from Fudan University, Shanghai, China, in 2012. Currently, he is working toward the M.E. degree in the Globa Information and Telecommunication Studies, Waseda University, Tokyo, Japan. His present research emphasizes on the study of energy efficiency problems in the ad hoc networks.

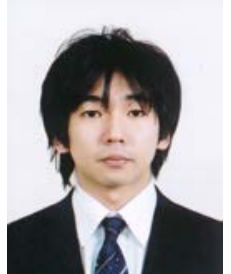

Ryo Yamamoto received his B.E. and M.E. degree in electronic information systems from Shibaura Institute of Technology, Tokyo, Japan, in 2007 and 2009. He received D.S. in global telecommunication studies from Waseda University, Tokyo, Japan, in 2013. He is presently a research associate of Global Information and Telecommunication Institute, Waseda University. He received the IEICE young researcher's award in 2010. His current research interests are mobile ad hoc networks and cross-layered protocols.

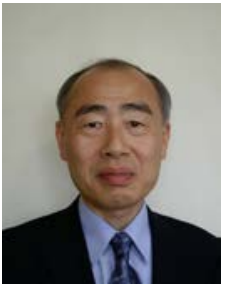

Yoshiaki Tanaka received the B.E., M.E., and D.E degrees in electrical engineering from the University of Tokyo, Tokyo, Japan, in 1974, 1976, and 1979, respectively. He became a staff at Department of Electrical Engineering, the University of Tokyo, in 1979, and has been engaged in teaching and researching in the fields of telecommunication networks, switching systems, and network security. He was a guest professor at Department of Communication Systems, Lund Institute of Technology, Sweden, from 1986 to 1987. He was also a visiting researcher at Institute for Posts and Telecommunications Policy, from 1988 to 1991, and at Institute for Monetary and Economic Studies, Bank of Japan, from 1994 to 1996 . He is presently a professor at Global Information and Telecommunication Institute, Waseda University, and a visiting professor at National Institute of Informatics. He received the IEEE Outstanding Student Award in 1977, the Niwa Memorial Prize in 1980, the IEICE Achievement Award in 1980, the Okawa Publication Prize in 1994, the TAF Telecom System Technology Award in 1995 and in 2006, the IEICE Information Network Research Award in 1996, in 2001, in 2004, and in 2006, the IEICE Communications Society Activity Testimonial in 1997 and in 1998, the IEICE Switching System Research Award in 2001, the IEICE Best Paper Award in 2005, the IEICE Network System Research Award in 2006, in 2008, and in 2011, the IEICE Communications Society Activity Award in 2008, the Commendation by Minister for Internal Affairs and Communications in 2009, the APNOMS Best Paper Award in 2009 and in 2012, and the IEICE Distinguished Achievement and Contributions Award in 2013. He is a Fellow of IEICE. 